\title{
Index of Subjects
}

Aeromonas, adhesion to. sp. to cell lines used as models for intestinal adhesion. 465

Aeromonas. distribution of, phenospecies and genospecies among strains isolated from water. foods or from human clinical samples. 39

Amblyomma hebraeum. laboratory reared, and Amblyomma cariegatum ticks differ in their susceptibility to infection with Coudria ruminantium. 345

Amblyomma variegatum, laboratory reared Amblyomma hebraeum and. ticks differ in their susceptibility to infection with Coudria ruminantium. 345

Antibiotic resistant. control of infection with multiple. bacteria in a hospital renal unit: the value of plasmid characterization. 61

$\beta$-Lactam resistance in normal faecal flora from South Africa. 243

Burkholderia pseudomallei, RAPD analysis of isolates of. from patients with recurrent melioidosis. 115

Campylobacter. effect of changes in processing to improve hygiene control on contamination of poultry carcasses with. $49 \tilde{5}$

Campylobacter jejuni, comparison of $\mathrm{PFGE}$, ribotyping and phage-typing in the epidemiological analysis of. serotype HS2 infections. 215

Campylobacter. the Public Health Laboratory Service national case-control study of primary indigenous sporadic cases of. infection, 15

Campylobacter. the speciation and subtyping of, isolates from sewage plants and waste water from a connected poultry abattoir using molecular techniques. 485

Canoeists. a serosurvey of water-borne pathogens amongst. in South Africa. 299

Coudria ruminantium, laboratory reared $A$ mblyommas hebraeum and Amblyomma rariegatum ticks in their susceptibility to infection with. 345

Cowpox. serological evidence for the reservoir hosts of. virus in British wildlife. 185

Cryptosporidiosis, an outbreak of. associated with a resort swimming pool. 545

Cryptosporidiosis, an outbreak of. in South London: what value the $p$ value. 279

Cryptosporidiosis, an outbreak of waterborne, associated with a public water supply in the LK. 123

Cryptospioridiosis. outbreak of. associated with a disinfected groundwater supply. 5i5

Entamoeba histolytica. prevalence of antibodies against. in Mexico measured by ELISA. 535

Escherichia coli, characterization of verocytoxin-producing. 0157 isolates from patients with haemolytic uraemic syndrome in Western Europe. 1

Escherichia coli, isolation of enterotoxigenic. from British troops in Saudi Arabia. 455

Escherichia coli. the role of heightened surveillance in an outbreak of. $0157 . \mathbf{H 7} .447$

Faecal contamination on children's hands and environmental surfaces in primary schools in Leeds. 527

Foot-and-mouth disease. antigenic analysis of SAT 2 serotype. virus isolates from Zimbabwe using monoclonal antibodies. 193

Genital warts. hand-genital transmission of? An analysis of prevalence data. 169

Haemolytic uraemic syndrome, characterization of verocytotoxin-producing Escherichia coli 0157 isolates from patients with. in Western Europe. 1

Haemopohilus influenzae, epidemiology of invasive. infections in England and Wales in the prevaccination era $(1990-2) .89$

Hepatitis A. prevalence of, antibody among disadrantaged gypsy children in northern Spain. 157

HIV-1, inactivation of. by chemical disinfectants: sodium hypochlorite. 567

Influenza. analysis of. a virus reinfection in children in Japan during 198391.591 
Influenza, study of the effectiveness of, vaccination in the elderly in the epidemic of 1989-90 using a general practice database, 581

Jembrana. the transmission of, disease, a lentivirus disease of Bos javanicus cattle, 367

Legionella pneumophila. molecular chracterization of a virulence-associated epitope on the lipopolysaccharide of, serogroup 1,71

Legionella. relationship between, spp. and antibody titres at a therapeutic thermal spa in Portugal. 79

Iisteria. occurrence of. species in ready to eat foods, 519

Measles. interpretation of serological surveillance data for, using mathematical models: implications for vaccine strategy, 139

Measles, priorities in global, control: report of an outbreak in N'Djamena, Chad, 309

Measles vaccination policy, 603

Measles vaccine efficacy during an outbreak in a highly vaccinated population: Incremental increase in protection with age at vaccination up to 18 months, 315

Meningoococcal, epidemiologial investigation of an outbreak of, meningitis in Makkah (Mecea), Saudi Arabia. 1992. 399

Meningococcal. targeted vaccination with, polysaccharide vaccine in one district of the Czech Republic. 411

Oysters, an outbreak of viral gastroenteritis associated with adequately prepared, 163

Pandemics. virology research and virulent human, 377

Poliomyelitis in the Netherlands: a review of population immunity and exposure between the epidemics in 1978 and 1992, 289

Rattus noriegicus. cryptosporidiosis reservoir in wild brown rats, in the CK, 207

Rickettsial. evidence for a high prevalence of spotted fever group, infections in diverse ecological zones of Inner Mongolia, 177

Salmonella enteritidis, chaos under canvas: a, PT $6 \mathrm{~B}$ outbreak, 513

Salmonella outbreak from microwave cooked food, 227

Salmonellosis, a pseudo-outbreak of, 31

Salmonellosis, nationwide outbreak of human, in Germany due to contaminated paprika and paprika-powdered potato chips, 501

Schistosoma mansoni. the development of an age structured model for schistosomiasis transmission dynamies and control and its validation for, 325

Staphyloroccus aureus, analysis of methicillin-resistant and methicillin-susceptible, by a molecular typing method based on coagulase gene polymorphisms, 419

Staphylocorcus aureus, carriage of, among 104 healthy persons during a 19-month period, 51

Streptococcal. a cluster of cases of, necrotizing fasciitis in Gloucestershire, 387

streptococrus pyogenes. clonal structure of invasive, in Northern Scotland, 231

Tick-borne encephalitis, roe deer as sentinels for endemicity of, virus, 355

Trimethoprim. prevalence and genetic location of non-transferable, resistant dihydrofolate reductase genes in South African commensal faecal isolates, 255

Tuberculosis in Inner London: evidence for an increase in young adults and immigrants, 133

Typhoid. infecting dose and severity of: analysis of volunteer data and examination of the influence of the definition of illness used, 23

Vibrio cholerae. an epidemiological study of, 01 in the Australian environment based on rRNA gene polymorhisms. 435

Vibrio cholerae. biotype traits and antibiotic susceptibility of, serogroup 01 before, during and after the emergence of the 0139 serogroup, 427

Viral gastroenteritis. an outbreak of, associated with adequately prepared oysters, 163

Whooping cough. application of pulsed field gel electrophoresis to the 1993 epidemic of, in the CK. 101 